\title{
Use of imaging as staging and surgical planning for pancreatic surgery
}

\author{
Andrew Hieu Nguyen, Laleh G. Melstrom \\ Department of Surgery, City of Hope, Duarte, CA, USA \\ Contributions: (I) Conception and design: All authors; (II) Administrative support: None; (III) Provision of study material or patients: None; (IV) \\ Collection and assembly of data: AH Nguyen; (V) Data analysis and interpretation: AH Nguyen; (VI) Manuscript writing: All authors; (VII) Final \\ approval of manuscript: All authors. \\ Correspondence to: Dr. Laleh G. Melstrom, MD. City of Hope National Medical Center, 1500 Duarte Road, Duarte, CA 91010, USA. \\ Email: lmelstrom@coh.org.
}

\begin{abstract}
Importance: In the past few decades, there has been rapid advancements in imaging technologies that have become irreplaceable in the pre-operative assessment of patients with pancreatic tumors. Modern imaging modalities, including computed tomography (CT) and endoscopic ultrasound (EUS), can provide critical information of the absence or presence of metastatic disease in pancreatic cancer, as well as details on the local extent and resectability, allowing for the selection of stage appropriate treatments and pre-operatively determined surgical approach.

Objective: The aim of this review is to discuss staging, resectability, and imaging for patients with pancreatic tumors.

Evidence Review: A literature review was performed of articles relevant to the topics of staging, resectability, and imaging of pancreatic tumors. Imaging modalities included CT, EUS, magnetic resonance imaging (MRI), positron emission tomography (PET), antibody-based and narrow band imaging.

Findings: CT pancreas protocol combined with EUS serve as the primary modalities in diagnosis, staging, and surgical planning in patients with pancreatic tumors. MRI is an alternative to CT with near equivalent utility in the pre-operative setting. In some circumstances, PET-CT may be a cost-effective initial study to detect distant disease.

Conclusions and Relevance: Current imaging technologies play a critical role in the evaluation of patients with pancreatic tumors. Advances in the past 3 decades in imaging technologies have revolutionized the process of assessment of stage and resectability in patients with pancreatic tumors. Future imaging technologies will address current limitation in the evaluation of occult metastatic disease.
\end{abstract}

Keywords: Magnetic resonance imaging (MRI); computed tomography (CT); pancreatic cancer; positron emission tomography (PET); endoscopic ultrasound

Submitted Mar 08, 2019. Accepted for publication May 08, 2019.

doi: 10.21037/hbsn.2019.05.04

View this article at: http://dx.doi.org/10.21037/hbsn.2019.05.04

\section{Introduction}

Over the past few decades, advances in technology and the methods in which abdominal imaging is interpreted have changed the way that we evaluate and treat patients with pancreatic cancer. Cross sectional imaging with computed tomography (CT), magnetic resonance imaging (MRI) and positron emission tomography (PET)-CT has largely abolished "exploratory surgery" as most stage IV metastatic disease to the liver can be determined in the radiology suite rather than the operating room. For the patients with resectable disease, pre-operative imaging allows surgeons to finely tailor a resection for a tumor of a specific location, adjacent to vital abdominal vascular structures. The days of mobilizing the duodenum to palpate a posteriorly located 


\begin{tabular}{ll} 
Table 1 AJCC TNM staging definitions $\left(8^{\text {th }}\right.$ Edition $)$ \\
\hline Stage & Description \\
\hline T0 & No evidence of primary tumor \\
T1 & Maximum tumor diameter $\leq 2 \mathrm{~cm}$ \\
T2 & Maximum tumor diameter $>2$ and $\leq 4 \mathrm{~cm}$ \\
T3 & Maximum tumor diameter $>4 \mathrm{~cm}$ \\
T4 & Tumor involves the celiac axis, SMA, and/or CHA \\
N & \\
N0 & No regional lymph node metastases \\
N1 & Metastasis in 1-3 regional lymph nodes \\
N2 & Metastasis in $\geq 4$ regional lymph nodes \\
M & \\
M0 & No distant metastases \\
M1 & Distant metastasis \\
\hline
\end{tabular}

Table 2 AJCC prognostic staging groups ( $8^{\text {th }}$ Edition)

\begin{tabular}{lccc}
\hline Stage & T & N & M \\
\hline Stage IA & T1 & N0 & M0 \\
Stage IB & T2 & N0 & M0 \\
Stage IIA & T3 & N0 & M0 \\
Stage IIB & T1-3 & N1 & M0 \\
Stage III & T1-3 & N2 & M0 \\
& T4 & Any N & M0 \\
Stage IV & Any T & Any N & M1 \\
\hline
\end{tabular}

tumor relative to the superior mesenteric artery as the primary means of assessing resectability are over, as the imaging of localized pancreatic tumors can reliably present the tumor's relationship to vascular structures and describe this critical interface. In this review, we discuss the role of imaging in staging and surgical planning and then the current imaging modalities that are primarily involved in these assessments in addition to novel imaging techniques on the horizon.

We present the following article in accordance with the Narrative Review reporting checklist (available at http:// dx.doi.org/10.21037/hbsn.2019.05.04).

\section{Staging}

Critical in the decision-making algorithm in pancreatic cancer is clinical staging, which is determined by physical exam, cross sectional imaging and often, endoscopic ultrasound with biopsy. Clinical staging is performed using the tumor node metastases (TNM) staging system as defined by the American Joint Committee on Cancer (AJCC) (Tables 1,2). The TNM system stratifies extent of disease based on $\mathrm{T}$, the tumor size and local extent, $\mathrm{N}$, involvement of regional lymph nodes, and $M$, the absence or presence of distant metastatic disease. AJCC staging for pancreatic cancer has recently undergone revision with the $8^{\text {th }}$ edition, published in 2017 (1). In the newest edition, T1 through T3 stages are based on tumor size, where $\mathrm{T} 1$ are tumors less than or equal to $2 \mathrm{~cm}, \mathrm{~T} 2$ are tumors greater than $2 \mathrm{~cm}$ and less than or equal to $4 \mathrm{~cm}$, and T3 are tumors greater than $4 \mathrm{~cm}$ in size. T4 tumors are those that involve the celiac axis or superior mesenteric artery. In the AJCC $7^{\text {th }}$ edition, T3 tumors were those that extended beyond the boundaries of the pancreas without celiac axis or superior mesenteric artery involvement. In the $8^{\text {th }}$ edition, there was additionally a revision of $\mathrm{N}$ staging. While in the $7^{\text {th }}$ edition, nodal status was staged dichotomously as $\mathrm{N} 0$ represented no involved regional lymph nodes and N1 was applied to disease involving any regional lymph nodes, the $8^{\text {th }}$ edition divides lymph node involvement into three categories, which may better stratify patients, as the number of lymph nodes involved has been associated with worse survival (2). AJCC $8^{\text {th }}$ edition defines $\mathrm{N} 0$ as no regional lymph nodes involved, N1 as 1 to 3 lymph nodes involved, and $\mathrm{N} 2$ as greater than or equal to 4 lymph nodes involved. In the $8^{\text {th }}$ edition, $M$ staging remains the same, simply, M0, without evidence of metastatic disease, and M1, with metastatic disease to distant lymph nodes or organs. Most commonly, metastatic disease is observed in the liver $(80 \%)$, but occasionally, there may also be disease located in the lungs, bones, adrenal glands and on the peritoneum (3). Thus, liver lesions in patients with pancreatic cancer should raise suspicion of M1 disease. With regards to lung lesions in patients with resectable pancreatic adenocarcinoma, up to $18 \%$ of patients may have small $(<1 \mathrm{~cm})$ pulmonary nodules and $90 \%$ of these lesions are benign and thus are of lesser significance than those lesions identified in the liver (4). The identification of M1 disease is associated with a particularly dismal prognosis. 

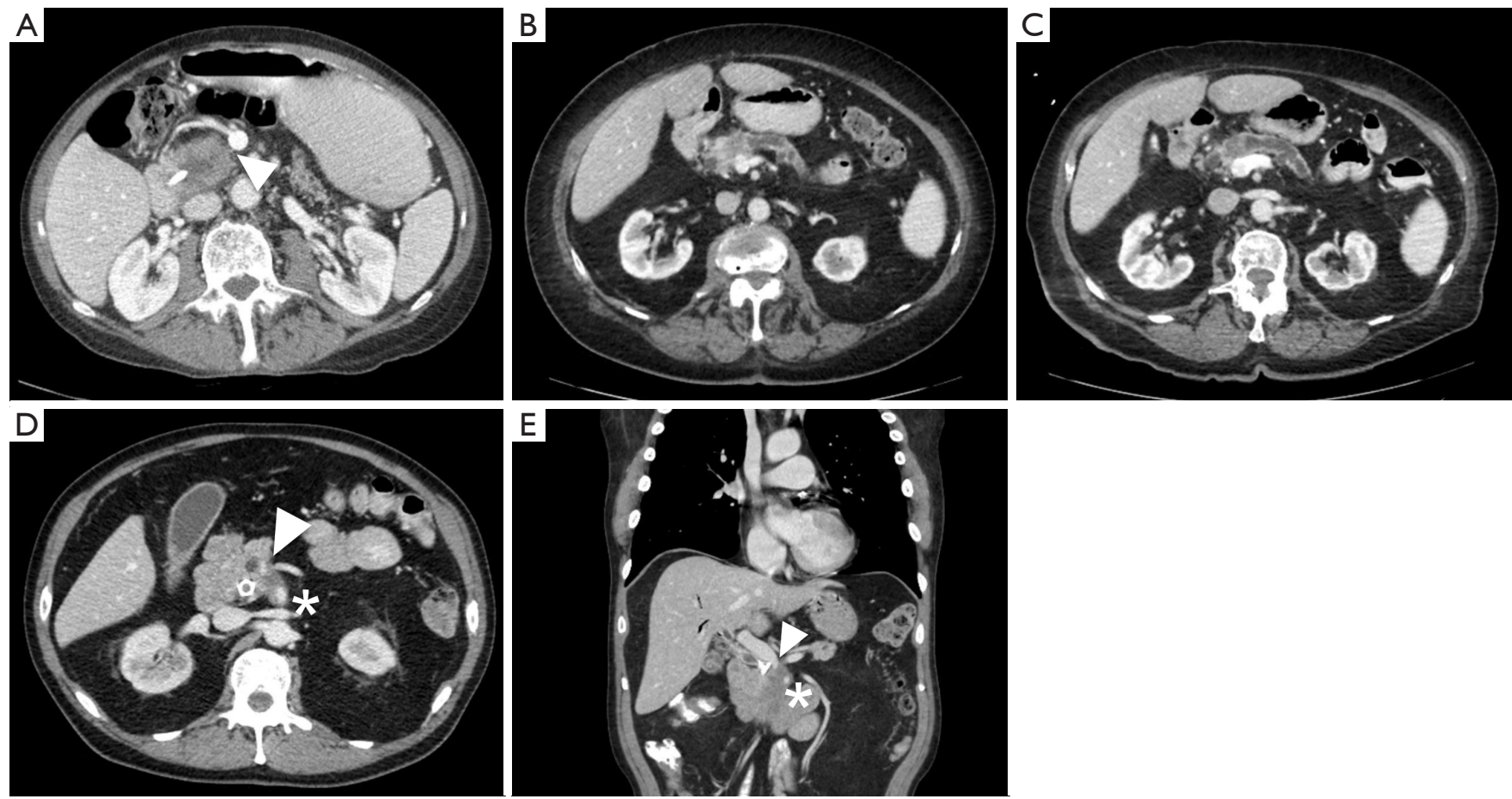

Figure 1 CT images of resectable, borderline resectable before and after treatment with neoadjuvant therapy and locally advanced pancreatic cancers. (A) Resectable pancreatic head mass with a clear fat plane (arrow) separating tumor from the superior mesenteric vein; (B) borderline resectable pancreatic head mass with tumor in contact with the superior mesenteric vein with slight distortion of the vessel. Note the distal pancreatic duct appears dilated; (C) borderline resectable pancreatic head mass following neoadjuvant chemotherapy with continued abutment and a distortion of a portion of the superior mesenteric vein; (D,E) locally advanced pancreatic head mass with encasement of the superior mesenteric vein (arrow) and superior mesenteric artery (star).

\section{Definitions of resectability}

While over half of patients with a diagnosis of pancreatic cancer present with distant disease and do not benefit from surgical resection, for patients with less extensive disease, the possibility of a successful resection is determined by careful interpretation of abdominal imaging studies (5). Cross sectional imaging allows anatomic localization of the tumor, particularly in relation to the superior mesenteric and portal veins (Figure 1). Tumors to the left of the vein are typically treated with distal pancreatectomy, whereas right-sided tumors are treated with a pancreaticoduodenectomy. Rarely, in synchronous pancreatic lesions in the head and body/ tail or in the situation of main duct intraductal papillary mucinous neoplasm (IPMN) involving the complete gland, total pancreatectomy may be the best-suited operation.

Generally, resectable tumors are those where it appears to be technically feasible to completely remove tumor and achieve microscopically uninvolved surgical margins. Resectable tumors are defined to be localized, non- metastatic tumors that have no extension of tumor to any of the arterial structures (superior mesenteric artery, celiac axis, or common hepatic artery) and no abutment, distortion, encasement, or occlusion of the superior mesenteric vein and portal vein, and a normal appearing fat plane separating tumor from vascular structures (Figure 1A) (6). In nonmetastatic disease, locally advanced tumors are those that are unresectable due to arterial or venous involvement without any technically feasible options for resection and reconstruction (Figure 1D,E) (7). In between resectable and locally advanced unresectable tumors, there is a group of tumors identified as borderline resectable, where certain imaging findings describe an intimate but limited anatomic relationship between tumor and the adjacent arterial and venous structures and where pre-operative radiation or chemotherapy may increase the odds of a negative surgical margin (Figure 1B,C).

Advances in cross sectional imaging, refinements of surgical technique, particularly in vascular reconstruction, 
and the use of neoadjuvant therapy have expanded the proportion of patients with pancreatic cancer who may be candidates for curative intent surgical resection. Historically, resectable tumors were limited to non-metastatic tumors distinctly separate from arterial and venous vasculature and it was only during the 1990s that it was shown that patients who underwent pancreaticoduodenectomy with a venous resection and negative margins achieved equivalent survival to those without a venous resection (8).

The degree of tumor contact with vascular structures has been found to contribute to recurrence and survival and thus, has importance in determining resectability. In a retrospective study of Japanese patients from 2001-2012 who had not received any pre-operative therapy, it was observed that survival was similar among patients who were identified on pre-operative imaging to be resectable without vascular contact and those with resectable tumors with less than $180^{\circ}$ contact with the portal or superior mesenteric vein with a median overall survival of about 2 years. Although, the patients without any vascular contact had a lower rate of positive surgical margin. For patients with tumor contact with the portal or superior mesenteric vein of greater than $180^{\circ}$, median overall survival falls to 17 months and for patients with contact with the superior mesenteric or hepatic artery, median overall survival falls to 11 months (9). In patients with arterial contact, a positive surgical margin was present in $50 \%$ of cases. These two later groups of patients are now included in "borderline" resectable pancreatic cancers, where the poorer survival and elevated rates of $\mathrm{R} 1$ resection with upfront surgery often directs patients towards pre-operative therapies. Recently, with modern treatment strategies such as pre-operative FOLFIRINOX chemotherapy plus chemoradiation, for patients who undergo a curative intent resection, $96 \%$ of patients may achieve an $\mathrm{R} 0$ resection with a 2 -year overall survival of greater than $70 \%(10)$.

In the evaluation of pancreatic tumor, borderline resectable is assigned based on the relation of tumor to the adjacent arterial and venous structures: the superior mesenteric vein, the portal vein, the superior mesenteric artery, the common hepatic artery, and the celiac axis. The criteria of borderline resectable have undergone multiple revisions through different research and consensus groups (Table 3). In 2006, a multidisciplinary group from M. D. Anderson Cancer Center published a definition of borderline resectable pancreatic cancer allowing for short segment occlusion of the superior mesenteric vein and portal vein with suitable proximal and distal vessel for resection and reconstruction, $<180^{\circ}$ abutment of tumor of the superior mesenteric artery, and short segment encasement or abutment of the common hepatic artery with sufficient proximal and distal vessel for resection and reconstruction (11). In 2008, a consensus committee that was co-sponsored by M. D. Anderson Cancer Center, and included members from the Americas Hepato-PancreatoBiliary Association, the Society of Surgical Oncology, and the Society for Surgery of the Alimentary Tract defined borderline resectable to standardize criteria for the purpose of multi-institutional research and allow comparison among multiple studies (12). These criteria were largely similar to those initially described in 2006. In 2013, a multi-institutional clinical trial, sponsored by the Alliance for Clinical Trials in Oncology, led by M. D. Anderson Cancer Center, and involving 14 different sites, defined again, essentially the same criteria for borderline resectable pancreatic cancer, but also including tumors with $<180^{\circ}$ interface with the celiac axis (13).

In 2016, the International Association of Pancreatology (IAP) also defined a set of anatomic criteria for borderline resectable pancreatic cancer following an internationally attended conference in Sendai, Japan (14). These criteria, largely adapted from the Japanese Pancreas Society classification subdivided anatomic borderline resectable pancreatic cancer to those with arterial involvement (BR-A) and those with venous involvement (BR-V), with the BR-A tumors being associated with poorer prognosis. These criteria remove subjective terminology such as abutment, as well as phrases such as "allowing for safe and complete resection and vein reconstruction", which relies heavily on the interpreting radiologist or surgeon. The IAP criteria allows $<180^{\circ}$ contact with the superior mesenteric artery and celiac axis, limited contact with the common hepatic artery, and $>180^{\circ}$ contact with the superior mesenteric and portal veins without exceeding the inferior border of the duodenum.

The National Comprehensive Cancer Network (NCCN) in their guidelines for the management of pancreatic adenocarcinoma, in a committee of 34 experts from 29 different institutions and 9 different specialties also define criteria for borderline resectable, resectable, and unresectable pancreatic cancer (15). Resectable pancreatic cancer has no arterial tumor contact and ideally, no tumor contact with the superior mesenteric vein or portal vein but allows for less than or equal to 180 degree contact without vein contour irregularity. In regards to tumor involvement with arterial structures, the definition of borderline 
Table 3 Criteria for borderline resectable pancreatic cancer

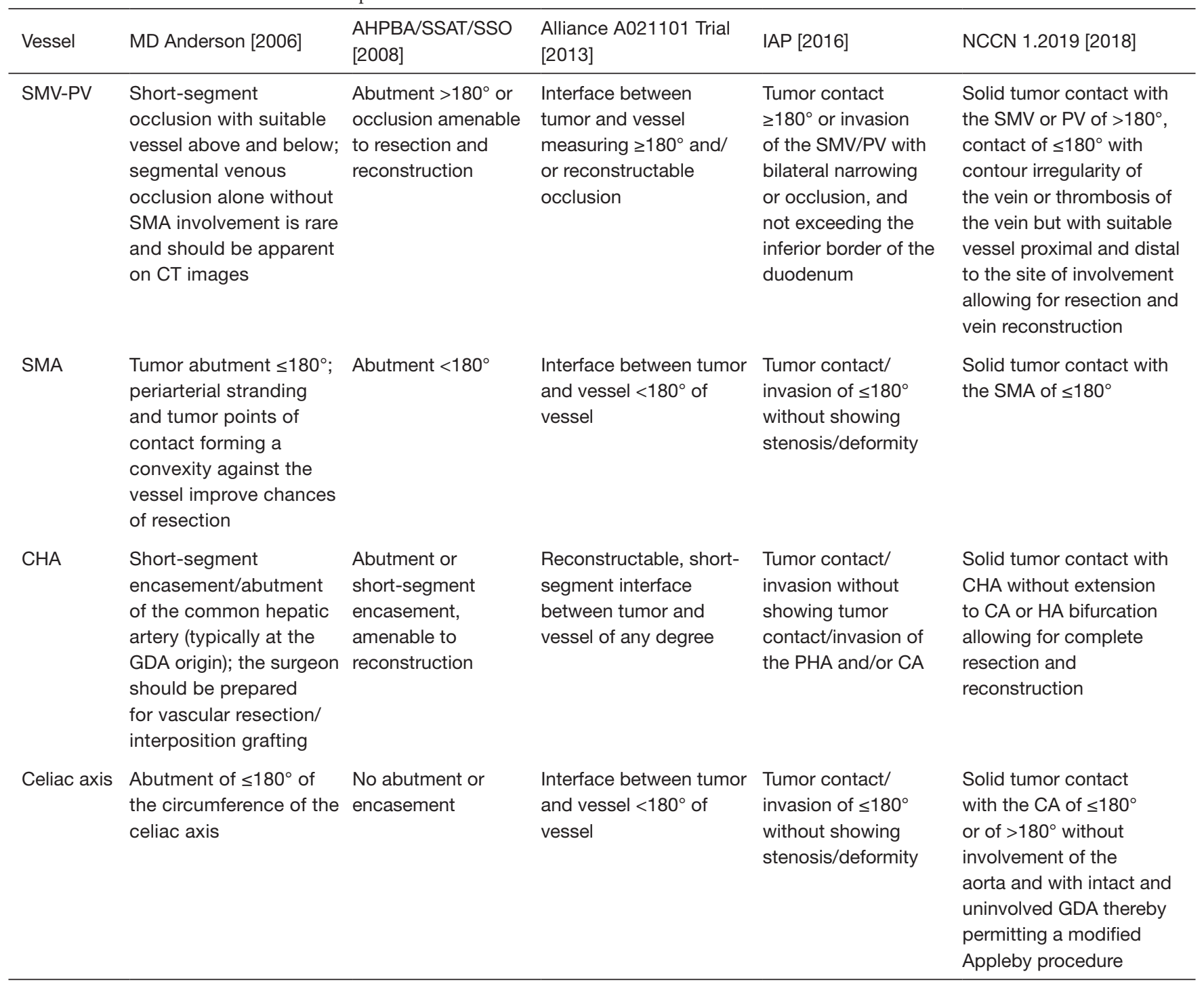

resectable allows for solid tumor contact with the common hepatic artery without extension to the celiac axis or hepatic artery bifurcation allowing for safe and complete resection and reconstruction, $\leq 180^{\circ}$ solid tumor contact to the superior mesenteric artery, or solid tumor contact to variant anatomy. For left-sided tumors, NCCN guidelines for borderline resectable tumors allow for solid tumor contact with the celiac axis of $\leq 180^{\circ}$ or if $>180^{\circ}$, must be without involvement of the aorta with an intact and uninvolved gastroduodenal artery, allowing for the modified Appleby procedure (although some committee members considered these tumors involving the celiac axis to be unresectable). In regards to tumor involvement with venous structures, the definition of borderline resectable allows for solid tumor contact with the superior mesenteric vein or portal vein of $>180^{\circ}$ or $\leq 180^{\circ}$ with vein contour irregularity or thrombosis with suitable proximal and distal vessel for resection and reconstruction, as well as solid tumor contact with the inferior vena cava. The NCCN categorizes unresectable tumors as those with distant metastasis, including nonregional lymph nodes. In addition, right-sided pancreatic tumors with solid tumor contact of $>180^{\circ}$ to the superior mesenteric artery or celiac access and left-sided tumors with solid tumor contact of $>180^{\circ}$ with the superior mesenteric artery or celiac axis or solid tumor contact with the celiac axis with aortic involvement are also considered 
Table 4 Indications and costs of imaging studies

\begin{tabular}{llr}
\hline Imaging technique & & Indication \\
\hline CT & For detection, staging, surgical planning & Estimated cost $^{\dagger}$ \\
EUS & For detection, diagnostic biopsy & $\$ 346$ \\
MRI & For detection, staging, surgical planning, evaluation of ductal anatomy (used especially in patients \\
& allergic to iodinated contrast, renal failure) & $\$ 569$ \\
PET-CT & For staging, especially in high risk patients for metastatic disease
\end{tabular}

${ }^{\dagger}$, costs from outpatient radiology and procedural Medicare average payments from http://www.cms.gov/.

unresectable. Unresectable venous involvement includes tumors involving the superior mesenteric vein or portal vein where reconstruction is not feasible or where tumor contacts the most proximal draining jejunal branches into the SMV for right-sided tumors.

\section{CT}

High-quality cross sectional imaging is critical and irreplaceable in the evaluation of patients with pancreatic tumors. Modern CT technology allows reliable assessment of the extent of disease and the relation of tumor to critical vascular structures at reasonable cost relative to other modalities (Table 4). Multidisciplinary teams can then accurately determine the best treatments for stage and in non-metastatic tumors, resectability and the potential technical challenges of an operation. The ability to optimally visualize a tumor of the pancreas is highly dependent on the protocol used, as a non-contrast CT scan is nearly useless in evaluating a pancreatic tumor and even in a venous phase CT study, a pancreatic adenocarcinoma may fade in with the rest of the parenchyma. In patients with a suspected or new diagnosis of pancreatic tumor with only prior suboptimal imaging, new, dedicated pancreatic protocol CT imaging should be performed.

Standard imaging for a pancreatic tumor is with a multiphase, pancreatic protocol with images acquired with a multi-detector CT scanner. Typically, $100-150 \mathrm{~mL}$ of iodinated contrast are injected at a rate of $3-5 \mathrm{~mL} / \mathrm{sec}$ and depending on institutional protocols, two or three phases are captured. In the past, pancreatic protocols were performed in three phases: with an early arterial phase, captured around 20 seconds, followed by a late arterial/pancreatic phase at 35-45 seconds, and then followed by a portal venous phase at about $55-65$ seconds $(16,17)$. With bolus tracking, where image acquisition times are adjusted based on contrast delivery to the aorta, time delays are shorter.
The early arterial phase has largely gone out of favor as the late arterial/pancreatic phase usually has excellent contrast enhancement of arterial structures, especially with maximum intensity projection images, allowing identification of anomalous anatomy as well as evaluation of the interface between tumor and arterial structures. High quality arterial/pancreatic phase images have limited the use of CT angiography and conventional angiography to define arterial anatomy in pancreatic tumors. The late arterial/ pancreatic phase is critical in assessing pancreatic tumors as it allows the sometimes subtle discrimination of a pancreatic tumor from normal parenchyma where adenocarcinoma will appear lower enhancing compared to adjacent parenchyma and neuroendocrine tumor will appear bright. The portal venous phase highlights the tumors relationship to the superior mesenteric and portal vein as well as the presence of hepatic metastases. While most protocols are dual phase, some have suggested that a single phase intermediate to the late arterial/pancreatic phase and portal venous phase at 50 seconds is sufficient to assess resectability of pancreatic tumors, while reducing radiation and data storage from multiphase protocols (18). Thin slices of at least $2 \mathrm{~mm}$ should be acquired for the pancreatic phase and $5 \mathrm{~mm}$ slices are reasonable for the portal venous phase images.

CT is an ideal study to assess anatomy around the pancreatic tumor with a spatial resolution up to half a millimeter (19). The data derived from an adequate study provides the surgeon a sense of the anatomic challenges to be encountered in the operating room. The celiac axis and superior mesenteric artery should be clearly visualized on the arterial phase, as well as the secondary and tertiary branches. Anomalous arterial anatomy in the forms of replaced or accessory hepatic arteries should also be easily identifiable. The superior mesenteric and portal veins become apparent on the venous phase, and particular attention can be placed on the interface between the tumor and venous structures as well as the proximal 

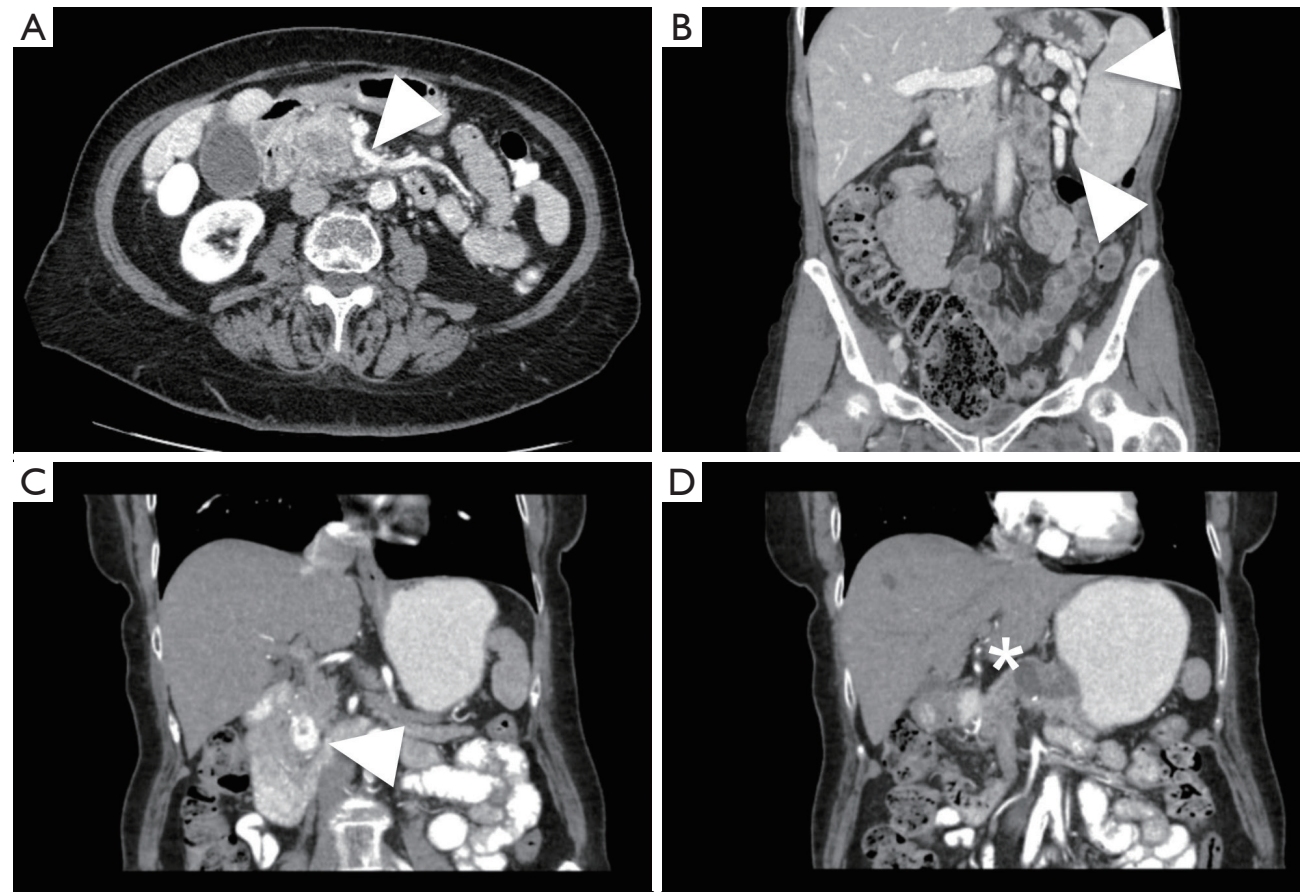

Figure 2 Various pre-operative findings that affect operative strategy. (A) Pancreatic head mass with adjacent large first jejunal branch (arrow) from superior mesenteric vein; (B) pancreatic body mass with splenic vein thrombosis and perisplenic varices (arrows); (C,D) arterial enhancing neuroendocrine tumor located in the uncinate process (arrow) with a non-enhancing pancreatic body cystadenoma (star) in the same patient.

jejunal superior mesenteric vein branches that can be a source of troublesome intra-operative bleeding (Figure $2 A$ ). The presence of a fat plane on CT images separating the tumor from vascular structures is associated with a $95 \%$ rate of successful resection without the need for vascular resection (20). Alternatively, when this fat plane is no longer present, certain vessel deformities may suggest a more intimate relationship between tumor and vein. These findings would include vessel flattening, vessel wall irregularity, tear drop or bird's beak shape, vessel caliber stenosis, or occlusion (21-23). As a result of venous occlusions, left-sided portal hypertension and gastric varices may occur with tumors of the body and tail (Figure 2B), or cavernous transformation of the portal vein may occur in tumors of the pancreatic head and uncinate process. CT will also identify situations where multiple pancreatic lesions may be identified in the same patient, requiring consideration of slightly more extensive resection than simply a pancreaticoduodenectomy or distal pancreatectomy (Figure 2C,D).

While high-resolution CT imaging is best in the assessment of tumor with respect to arterial and venous anatomy, it still has certain drawbacks. Although the portal venous phase facilitates detection of liver metastases, the sensitivity is only about $70-75 \%$, and is even less for hepatic metastases less than $1 \mathrm{~cm}$ in size $(24,25)$. CT findings have been found to poorly predict lymph node metastases, even when details such as morphology, size, clustering, and absence of a fatty hilum are characterized (26). In one study, at an optimal time within 25 days prior to surgery, CT imaging has a sensitivity of only $85 \%$ to detect metastases (27). The sub-optimal sensitivity in detecting lymph node and liver metastases reduces the overall preoperative accuracy of CT staging of nodal and metastases. Thus, even with recently performed imaging, laparoscopy has utility to evaluate for metastatic disease not visualized on pre-operative imaging (25). For borderline patients that had undergone neoadjuvant therapy, restaging CT scan is often performed, yet very few patients are downstaged in imaging. In a study of borderline resectable pancreatic cancers who received neoadjuvant therapy, less than $1 \%$ of patients were radiographically down staged to resectable status, although $95 \%$ of patients in this study did achieve R0 resection (28). CT imaging has poor sensitivity to truly 
predict resectability in the neoadjuvant setting as the pretherapy hazy interface between tumor and vascular structure suggestive of invasion is often replaced with post-therapy inflammation and fibrosis, which is indistinguishable from residual cancer at that margin on the subsequent CT images $(29,30)$. It has been suggested that following neoadjuvant therapy, observing "partial regression" of tumor contact with vascular structures to be associated with R0 resection (31). Despite these drawbacks, CT remains the primary imaging modality in staging and surgical planning in pancreatic surgery.

\section{Endoscopic ultrasound}

In the 1990s when pancreatic imaging by CT scan was maturing, endoscopic ultrasound with fine-needle aspiration (EUS-FNA) began its more widespread adoption as a key complementary study in the initial diagnosis and staging of pancreatic cancer. EUS of the pancreas is performed with an endoscope with typically a linear array ultrasound transducer tip with a working channel to deliver a 22 or 25 gauge FNA needle, where pancreatic lesions can be visualized either from the stomach or the duodenum. While transabdominal ultrasound is severely limited, as the ultrasonic signal from an external probe must traverse multiple organs to reach the pancreas, EUS allows acquisition of sonographic images from directly adjacent to the pancreas, within sufficient proximity to allow needle biopsy of even small pancreatic lesions. Transgastric views allow visualization of the pancreatic neck, body, and tail, as well as the splenic vein, celiac axis, common hepatic artery and superior mesenteric artery. Transduodenal views allow access to the pancreas head and uncinate process as well as the bile duct, portal vein and superior mesenteric vein. Pancreatic adenocarcinoma will often appear as a hypoechoic pancreatic mass with irregular borders on EUS. Additionally, adjacent regional lymph nodes can be evaluated and sampled as well with this technique. The transgastric view also allows accessibility and visualization of the left lateral segment of the liver as well.

In the context of diagnostic work-up, EUS-FNA is an excellent study with $82-96 \%$ sensitivity and $99-100 \%$ specificity $(32,33)$. Outside of the patients with prior bariatric surgery that may limit access, it is the best approach to acquire tissue for diagnosis and has high sensitivity, particularly with smaller pancreatic lesions (33). EUS has been found to have higher accuracy than CT or MRI in assessing tumor size as well as lymph node involvement (34).
While CT can provide a global view of anatomy with excellent resolution at the interface between tumor and vascular structures, EUS can similarly visualize this interface with high sensitivity, particularly along the portal and splenic veins (35). On EUS, irregularity of the venous wall, loss of interface between tumor and venous structures, vascular encasement or occlusion, obliteration of the vessel with the presence of collaterals, and close proximity to the tumor can be visualized (23). In contrast to venous assessment, arterial evaluation is more limited with sensitivity and specificity as low as $50 \%$ and $58 \%$, respectively (23).

\section{MRI}

MRI is another cross-sectional imaging modality used frequently in pancreatic imaging, especially with technical advancements that allow for improved spatial resolution and quicker acquisition times. MRI of the pancreas is acquired over a combination of pre-contrast T1-weighted, T2-weighted, and diffusion-weighted imaging sequences as well as post-contrast T1-weighted images, timed in a similar manner to contrast protocols of CT (36). In the pre-contrast images, T1-weighted images show the pancreas with high signal intensity, allowing assessment of parenchyma and thus, detection of pancreatic tumors, which for adenocarcinoma would appear as a focal low signal intensity. T2-weighted images allow for evaluation of the pancreatic and biliary ducts, which is particularly useful in cystic pancreatic disease or in tumors where ductal dilation can allow for identification of the point of duct obstruction. Magnetic resonance cholangiopancreatography (MRCP) reconstructions rely on these sequences. Diffusionweighted sequences can be useful in detecting pancreatic and liver lesions as well as abnormal lymph nodes. Similar to pancreatic protocol CT studies, a Gadolinium contrast enhancing sequence in the late arterial/pancreatic phase in a T1-weighted sequence will show pancreatic tumors in a similar fashion, where adenocarcinoma will appear as a hypoenhancing lesion and neuroendocrine tumors will appear as hyperenhancing relative to the surrounding parenchyma. Arterial and venous anatomy can be reliably evaluated on these contrast phases, providing nearly equivalent information compared to CT images.

While the spatial resolution of MRI is inferior to CT and occasionally lesions may measure smaller on MRI, the ability to assess pancreatic cancer for vascular involvement with contrast-enhanced MRI has been found to be equivalent to contrast-enhanced CT (37). Additionally, there are particular 
situations where MRI is superior and situations where MRI is indicated. Specific indications to acquire MRI over CT include patients with iodinated contrast allergy or renal failure, imaging performed in pregnant women or children where ionizing radiation is contraindicated, or where CT is unable to produce optimal images. For patients with renal failure and contrast is contraindicated, non-contrast MRI has far superior soft tissue discrimination compared to noncontrast CT. MRI has also been found to be superior to $\mathrm{CT}$ in the detection of liver metastases with a sensitivity of $90-93 \%$ (24). MRI is also better than CT at defining ductal anatomy with MRCP, without unnecessary exposure to ionizing radiation, making it particularly useful in the surveillance of patients with cystic disease.

\section{PET}

While most pancreatic cancers can be adequately staged and evaluated prior to surgery with EUS-FNA and CT, with the occasional need for MRI, PET-CT may be reserved for special conditions. PET-CT uses fluorine-18 fluorodeoxyglucose (FDG) as a glucose analog radiotracer preferentially taken up by hypermetabolic foci of tumor cells, relative to normal parenchyma, and the tracer is detected in cross sectional images and displayed superimposed over CT images. Pancreatic cancer will typically appear as having intense FDG uptake. While CT and MRI can distinguish fine local details in the direct vicinity of the primary tumor, PET-CT is an ideal imaging modality to detect nodal and distant metastases that may be missed using other modalities. PET-CT has a sensitivity of approximately $90 \%$ in detecting distant metastases, superior to CT or MRI $(38,39)$. Some have suggested PET-CT to be more accurate and more cost-effective than CT alone for initial staging. While relatively costly as an individual test, it may detect metastatic disease at initial imaging, preventing a futile and unnecessary attempt at resection in the operating room in up to $20 \%$ of patients $(40,41)$. However, while PET-CT has high sensitivity (97\%) in detecting metastases larger than $1 \mathrm{~cm}$ in diameter, sensitivity falls to $43 \%$ for smaller lesions (42).

Recently, PET-MRI has emerged as a modality combining PET with MRI, rather than CT. In a prospective study of patients with pancreatic tumors who received both a contrast enhanced PET-CT and a PET-MRI, PET-MRI was been found to have equivalent diagnostic performance in both staging as well as determining resectability (43). PET-MRI has the advantages of the absence of the radiation exposure of CT imaging as well as improved softtissue discrimination provided by MRI images. In patients who require re-staging imaging studies, PET-MRI limits radiation exposure, while enhancing the ability to detect distant disease and in patients who receive neoadjuvant therapy, treatment response may be observed in changes of metabolic signal by PET when the radiographic tumor size does not alter significantly (44).

\section{Future directions}

Technological advances in imaging have allowed physicians to accurately pre-operatively evaluate patients with pancreatic tumors. Unquestionably, in the near future, the current technologies will further improve with new protocols in image acquisition and post-processing, while other newer technologies develop. However, a particular challenge in imaging is identifying patients with stage IV disease with microscopic distant metastases, which is poorly addressed with current imaging modalities. The advancement of technologies to identify these patients will allow more accurate staging, improved selection of patients most appropriate for resection, and streamline care for patients with more advanced disease.

Antibody-based imaging is a strategy to gain specificity in localizing cancer cells that express specific antigens. CA19-9 is a common biomarker frequently expressed with pancreatic cancer. Modified antibodies against CA19-9 have been developed and studied in pre-clinical models. Theoretically, these antibodies can be conjugated to various markers, whether it is a radioisotope, a fluorescent marker, or a paramagnetic particle (45). In CA19-9 positive tumors, these modified antibodies may localize to tumor cells of the primary tumor as well as lymph node and distant metastatic sites. The diagnostic efficacy has been demonstrated in multiple in vivo mouse models of pancreatic cancer $(46,47)$. CA19-9 is considered a specific marker for pancreatic cancer but is expressed at increased levels in obstructive jaundice and is only expressed in $\sim 66 \%$ of patients with pancreatic cancer (48). However, in a patient with stable appearing disease on conventional imaging, absence of biliary obstruction, but a new elevation in CA19-9, antibody-based imaging may help identify the area of disease progression.

While the majority of late stage pancreatic cancers can be identified with non-invasive imaging modalities, still, diagnostic laparoscopy has an important role in identifying peritoneal disease and small liver lesions that are undetected 
with current imaging. For CT scans performed within 30 days of exploration, in $10-15 \%$ patients, metastases may be observed at exploration following a negative CT scan (27). Yet, the ability to detect peritoneal disease with laparoscopy alone is limited to macroscopically visible metastases, as $10 \%$ of patients undergoing curative resection may have positive peritoneal cytology by intraoperative peritoneal washings (49). These patients with positive peritoneal cytology have poorer prognosis compared to those without microscopic peritoneal disease (50). Narrow band imaging uses 415 and $540 \mathrm{~nm}$ light through the laparoscope that can allow better visualization of microvascular structures, enhancing the detection of microscopic peritoneal tumor deposits. Narrow band imaging in laparoscopy has been found to have improved sensitivity in detecting peritoneal metastases compared to white light imaging in gastric cancer, yet benefits have yet to be found in pancreatic cancer $(51,52)$. Similar to using narrow band imaging to enhance the accuracy of diagnostic laparoscopy, fluorophoreconjugated tumor specific antibodies have been proposed and tested in animal models to aide identification of subtle metastatic disease at laparoscopy (53).

\section{Conclusions}

In parallel with advances in the past three decades in surgical approach to pancreatic tumors, there have been significant advances in imaging technologies and the ways they are utilized. CT pancreas protocol combined with endoscopic ultrasound serve as the primary modalities in diagnosis, staging, and surgical planning in patients with pancreatic tumors. MRI is an alternative to CT with near equivalent utility in the pre-operative setting. In some circumstances, PET-CT may be a cost-effective initial study to detect distant disease.

\section{Acknowledgments}

Funding: None.

\section{Footnote}

Provenance and Peer Review: This article was commissioned by the Guest Editor (Dr. Yuman Fong) for the series "Imaging and HPB Surgery" published in Hepatobiliary Surgery and Nutrition. The article was sent for external peer review organized by the Guest Editor and the editorial office.
Reporting Checklist: The authors have completed the Narrative Review reporting checklist. Available at http:// dx.doi.org/10.21037/hbsn.2019.05.04

Conflicts of Interest: Both authors have completed the ICMJE uniform disclosure form (available at http://dx.doi. org/10.21037/hbsn.2019.05.04). The series "Imaging and HPB Surgery" was commissioned by the editorial office without any funding or sponsorship. The authors have no other conflicts of interest to declare.

Ethical Statement: The authors are accountable for all aspects of the work in ensuring that questions related to the accuracy or integrity of any part of the work are appropriately investigated and resolved.

Open Access Statement: This is an Open Access article distributed in accordance with the Creative Commons Attribution-NonCommercial-NoDerivs 4.0 International License (CC BY-NC-ND 4.0), which permits the noncommercial replication and distribution of the article with the strict proviso that no changes or edits are made and the original work is properly cited (including links to both the formal publication through the relevant DOI and the license). See: https://creativecommons.org/licenses/by-nc-nd/4.0/.

\section{References}

1. Allen PJ, Kuk D, Castillo CF, et al. Multi-institutional Validation Study of the American Joint Commission on Cancer (8th Edition) Changes for $\mathrm{T}$ and N Staging in Patients With Pancreatic Adenocarcinoma. Ann Surg 2017;265:185-91.

2. Murakami $Y$, Uemura K, Sudo T, et al. Number of metastatic lymph nodes, but not lymph node ratio, is an independent prognostic factor after resection of pancreatic carcinoma. J Am Coll Surg 2010;211:196-204.

3. Makohon-Moore A, Iacobuzio-Donahue CA. Pancreatic cancer biology and genetics from an evolutionary perspective. Nat Rev Cancer 2016;16:553-65.

4. Chang ST, Nguyen DC, Raptis C, et al. Natural history of preoperative subcentimeter pulmonary nodules in patients with resectable pancreatic adenocarcinoma: a retrospective cohort study. Ann Surg 2015;261:970-5.

5. Siegel RL, Miller KD, Jemal A. Cancer statistics, 2016. CA Cancer J Clin 2016;66:7-30.

6. Hong SB, Lee SS, Kim JH, et al. Pancreatic Cancer CT: Prediction of Resectability according to NCCN Criteria. 
Radiology 2018;289:710-8.

7. Abou-Khalil J, Rocha FG. Surgical strategies and novel therapies for locally advanced pancreatic cancer. J Surg Oncol 2017;116:16-24.

8. Lopez NE, Prendergast C, Lowy AM. Borderline resectable pancreatic cancer: definitions and management. World J Gastroenterol 2014;20:10740-51.

9. Murakami Y, Satoi S, Sho M, et al. National Comprehensive Cancer Network Resectability Status for Pancreatic Carcinoma Predicts Overall Survival. World J Surg 2015;39:2306-14.

10. Murphy JE, Wo JY, Ryan DP, et al. Total Neoadjuvant Therapy With FOLFIRINOX Followed by Individualized Chemoradiotherapy for Borderline Resectable Pancreatic Adenocarcinoma: A Phase 2 Clinical Trial. JAMA Oncol 2018;4:963-9.

11. Varadhachary GR, Tamm EP, Abbruzzese JL, et al. Borderline resectable pancreatic cancer: definitions, management, and role of preoperative therapy. Ann Surg Oncol 2006;13:1035-46.

12. Vauthey JN, Dixon E. AHPBA/SSO/SSAT Consensus Conference on Resectable and Borderline Resectable Pancreatic Cancer: rationale and overview of the conference. Ann Surg Oncol 2009;16:1725-6.

13. Katz MH, Shi Q, Ahmad SA, et al. Preoperative Modified FOLFIRINOX Treatment Followed by CapecitabineBased Chemoradiation for Borderline Resectable Pancreatic Cancer: Alliance for Clinical Trials in Oncology Trial A021101. JAMA Surg 2016;151:e161137.

14. Isaji S, Mizuno S, Windsor JA, et al. International consensus on definition and criteria of borderline resectable pancreatic ductal adenocarcinoma 2017. Pancreatology 2018;18:2-11.

15. Tempero MA, Malafa MP, Al-Hawary M, et al. Pancreatic Adenocarcinoma, Version 2.2017, NCCN Clinical Practice Guidelines in Oncology. J Natl Compr Canc Netw 2017;15:1028-61.

16. Bae KT. Intravenous contrast medium administration and scan timing at CT: considerations and approaches. Radiology 2010;256:32-61.

17. Lu DS, Vedantham S, Krasny RM, et al. Two-phase helical CT for pancreatic tumors: pancreatic versus hepatic phase enhancement of tumor, pancreas, and vascular structures. Radiology 1996;199:697-701.

18. Imbriaco M, Megibow AJ, Camera L, et al. Dualphase versus single-phase helical CT to detect and assess resectability of pancreatic carcinoma. AJR Am J Roentgenol 2002;178:1473-9.

19. Lin E, Alessio A. What are the basic concepts of temporal, contrast, and spatial resolution in cardiac CT? J Cardiovasc
Comput Tomogr 2009;3:403-8.

20. Loyer EM, David CL, Dubrow RA, et al. Vascular involvement in pancreatic adenocarcinoma: reassessment by thin-section CT. Abdom Imaging 1996;21:202-6.

21. Hough TJ, Raptopoulos V, Siewert B, et al. Teardrop superior mesenteric vein: CT sign for unresectable carcinoma of the pancreas. AJR Am J Roentgenol 1999;173:1509-12.

22. Li H, Zeng MS, Zhou KR, et al. Pancreatic adenocarcinoma: the different $\mathrm{CT}$ criteria for peripancreatic major arterial and venous invasion. $\mathrm{J}$ Comput Assist Tomogr 2005;29:170-5.

23. Buchs NC, Chilcott M, Poletti PA, et al. Vascular invasion in pancreatic cancer: Imaging modalities, preoperative diagnosis and surgical management. World J Gastroenterol 2010;16:818-31.

24. Motosugi U, Ichikawa T, Morisaka H, et al. Detection of pancreatic carcinoma and liver metastases with gadoxetic acid-enhanced MR imaging: comparison with contrast-enhanced multi-detector row CT. Radiology 2011;260:446-53.

25. Jimenez RE, Warshaw AL, Fernandez-Del Castillo C. Laparoscopy and peritoneal cytology in the staging of pancreatic cancer. J Hepatobiliary Pancreat Surg 2000;7:15-20.

26. Roche CJ, Hughes ML, Garvey CJ, et al. CT and pathologic assessment of prospective nodal staging in patients with ductal adenocarcinoma of the head of the pancreas. AJR Am J Roentgenol 2003;180:475-80.

27. Raman SP, Reddy S, Weiss MJ, et al. Impact of the time interval between MDCT imaging and surgery on the accuracy of identifying metastatic disease in patients with pancreatic cancer. AJR Am J Roentgenol 2015;204:W37-42.

28. Katz MH, Fleming JB, Bhosale P, et al. Response of borderline resectable pancreatic cancer to neoadjuvant therapy is not reflected by radiographic indicators. Cancer 2012;118:5749-56.

29. Morgan DE, Waggoner CN, Canon CL, et al. Resectability of pancreatic adenocarcinoma in patients with locally advanced disease downstaged by preoperative therapy: a challenge for MDCT. AJR Am J Roentgenol 2010;194:615-22.

30. Joo I, Lee JM, Lee ES, et al. Preoperative MDCT Assessment of Resectability in Borderline Resectable Pancreatic Cancer: Effect of Neoadjuvant Chemoradiation Therapy. AJR Am J Roentgenol 2018;210:1059-65.

31. Cassinotto C, Mouries A, Lafourcade JP, et al. Locally advanced pancreatic adenocarcinoma: reassessment of response with CT after neoadjuvant chemotherapy and 
radiation therapy. Radiology 2014;273:108-16.

32. Williams DB, Sahai AV, Aabakken L, et al. Endoscopic ultrasound guided fine needle aspiration biopsy: a large single centre experience. Gut 1999;44:720-6.

33. Gress FG, Hawes RH, Savides TJ, et al. Role of EUS in the preoperative staging of pancreatic cancer: a large single-center experience. Gastrointest Endosc 1999;50:786-91.

34. Soriano A, Castells A, Ayuso C, et al. Preoperative staging and tumor resectability assessment of pancreatic cancer: prospective study comparing endoscopic ultrasonography, helical computed tomography, magnetic resonance imaging, and angiography. Am J Gastroenterol 2004;99:492-501.

35. Brugge WR, Lee MJ, Kelsey PB, et al. The use of EUS to diagnose malignant portal venous system invasion by pancreatic cancer. Gastrointest Endosc 1996;43:561-7.

36. O'Neill E, Hammond N, Miller FH. MR imaging of the pancreas. Radiol Clin North Am 2014;52:757-77.

37. Arslan A, Buanes T, Geitung JT. Pancreatic carcinoma: MR, MR angiography and dynamic helical CT in the evaluation of vascular invasion. Eur J Radiol 2001;38:151-9.

38. Kauhanen SP, Komar G, Seppanen MP, et al. A prospective diagnostic accuracy study of $18 \mathrm{~F}$-fluorodeoxyglucose positron emission tomography/computed tomography, multidetector row computed tomography, and magnetic resonance imaging in primary diagnosis and staging of pancreatic cancer. Ann Surg 2009;250:957-63.

39. Heinrich S, Goerres GW, Schafer M, et al. Positron emission tomography/computed tomography influences on the management of resectable pancreatic cancer and its cost-effectiveness. Ann Surg 2005;242:235-43.

40. Ghaneh P, Hanson R, Titman A, et al. PET-PANC: multicentre prospective diagnostic accuracy and health economic analysis study of the impact of combined modality 18 fluorine-2-fluoro-2-deoxy-d-glucose positron emission tomography with computed tomography scanning in the diagnosis and management of pancreatic cancer. Health Technol Assess 2018;22:1-114.

41. Kim R, Prithviraj G, Kothari N, et al. PET/CT Fusion Scan Prevents Futile Laparotomy in Early Stage Pancreatic Cancer. Clin Nucl Med 2015;40:e501-5.

42. Fröhlich A, Diederichs CG, Staib L, et al. Detection of liver metastases from pancreatic cancer using FDG PET. J Nucl Med 1999;40:250-5.

43. Joo I, Lee JM, Lee DH, et al. Preoperative Assessment of Pancreatic Cancer with FDG PET/MR Imaging versus FDG PET/CT Plus Contrast-enhanced Multidetector
CT: A Prospective Preliminary Study. Radiology 2017;282:149-59.

44. Wang ZJ, Behr S, Consunji MV, et al. Early Response Assessment in Pancreatic Ductal Adenocarcinoma Through Integrated PET/MRI. AJR Am J Roentgenol 2018;211:1010-9.

45. Warram JM, de Boer E, Sorace AG, et al. Antibody-based imaging strategies for cancer. Cancer Metastasis Rev 2014;33:809-22.

46. Girgis MD, Federman N, Rochefort MM, et al. An engineered anti-CA19-9 cys-diabody for positron emission tomography imaging of pancreatic cancer and targeting of polymerized liposomal nanoparticles. J Surg Res 2013;185:45-55.

47. Houghton JL, Zeglis BM, Abdel-Atti D, et al. Sitespecifically labeled CA19.9-targeted immunoconjugates for the PET, NIRF, and multimodal PET/NIRF imaging of pancreatic cancer. Proc Natl Acad Sci U S A 2015;112:15850-5.

48. Berger AC, Garcia M, Jr., Hoffman JP, et al. Postresection CA 19-9 predicts overall survival in patients with pancreatic cancer treated with adjuvant chemoradiation: a prospective validation by RTOG 9704. J Clin Oncol 2008;26:5918-22.

49. Abe T, Ohuchida K, Endo S, et al. Clinical importance of intraoperative peritoneal cytology in patients with pancreatic cancer. Surgery 2017;161:951-8.

50. Oh SY, Edwards A, Mandelson MT, et al. Localized pancreatic cancer with positive peritoneal cytology as a sole manifestation of metastatic disease: a single-institution experience. Am J Surg 2017;213:94-9.

51. Kikuchi H, Kamiya K, Hiramatsu Y, et al. Laparoscopic narrow-band imaging for the diagnosis of peritoneal metastasis in gastric cancer. Ann Surg Oncol 2014;21:3954-62.

52. Schnelldorfer T, Jenkins RL, Birkett DH, et al. Laparoscopic narrow band imaging for detection of occult cancer metastases: a randomized feasibility trial. Surg Endosc 2016;30:1656-61.

53. Tran Cao HS, Kaushal S, Metildi CA, et al. Tumor-specific fluorescence antibody imaging enables accurate staging laparoscopy in an orthotopic model of pancreatic cancer. Hepatogastroenterology 2012;59:1994-9.

Cite this article as: Nguyen AH, Melstrom LG. Use of imaging as staging and surgical planning for pancreatic surgery. HepatoBiliary Surg Nutr 2020;9(5):603-614. doi: 10.21037/ hbsn.2019.05.04 